\title{
Concurrent Respiratory Disease in Broiler Chickens in Egypt during 2020
}

\author{
Nahed Yehia*, Fatma Amer, Abdelhafez Samir, Mohamed Samy, Ahmed Sedeek, Neveen Rebie, Wafaa \\ Mohammed, and Naglaa Hagag \\ Reference Laboratory for Veterinary Quality Control on Poultry Production, Animal Health Research Institute, Agriculture Research Center, Giza \\ 12618, Egypt \\ *Corresponding author's Email: nahedyehia@yahoo.com; (DORCiD: 0000-0002-2823-6467
}

\begin{abstract}
Poultry production has been affected by multiple respiratory diseases triggering serious economic losses in Egypt. The current study aimed to investigate the situation and genetic evolution of respiratory diseases in Egypt during 2020. A total of 53 samples were collected from infected flocks suffering from respiratory signs and variable mortality rates from nine governorates in Egypt during 2020. The collected samples were examined for the detection of respiratory disease viruses (Avian influenza virus (AIV (H5N8, H9N2), Infectious bronchitis virus (IBV), and Newcastle disease virus (NDV)) by rRT-PCR. The single infection was confirmed in $90.6 \%$ (37.7\% I.B, 30.2\% AIV (H5N8), 9.4\% I.B and 5.7\% NDV) and co-infection of HPAIV (H5N8) + I.BV and LPAIV (H9N2) +IBV were detected in $3.8 \%$ of nine governorates. The HA gene of HPAIV (H5N8) was cluster to clad 2.3.4.4.1b in a new branch with characteristic specific mutations especially in T140A in antigenic site A and R72S in the receptorbinding site, compared to A/duck/Egypt/F446/2017 with low A.A identity percent with vaccinal strains of H5N1 and H5N2 reaching to $91.9-94 \%$ and $84.6 \%$, respectively. The HA gene of AIV (H9N2) belonged to A/quail/Hong Kong/G1/97-like virus clustered with group B with a specific mutation (212I) that may affect the human transmission of the virus. The HVRs of S1 gene of IBV cluster to GI23 (Egy Var I) clad with multiple mutations in HVR1 and HVR2, compared to IBV/CU/4/2014 and low identity percent (68.3-78.8\%) with vaccine strains (H120, M41, 4/91). In conclusion, respiratory disease continues to circulate and rapidly evolve in Egypt during 2020.
\end{abstract}

Keywords: HPAIV (H5N8), IBV, Genetic characterization, LPAIV(H9N2), Respiratory disease

\section{INTRODUCTION}

The poultry industry is one of the most significant industries impacting the national economy in Egypt by promoting investment and jobs availability. Moreover, the primary sources of inexpensive animal protein for poor people in Egypt are poultry meat and eggs (Abdelwhab and Hafez, 2011).

Regrettably, the poultry industry in Egypt has been affected mainly by viral respiratory diseases for several years (Haghighat-Jahromi et al., 2008; Sediek, 2013; Awad et al, 2016). The inability to control this disease results in significant economic losses in the poultry sector. Avian influenza (highly pathogenic, low pathogenic), Newcastle Disease Virus (NDV) and Infectious Bronchitis Virus (IBV) alone or mixed infection (with each other or other bacterial infection) are the main cause of respiratory disease affect poultry farm with a high mortality rate (Radwan et al., 2013; Hassan et al., 2016; Samy and Naguib, 2018).

Avian Influenza (AI) was the most severe respiratory disease in Egypt. The Highly Pathogenic Avian Influenza (HPAI) H5N8 was first recorded in 2016 from a wild bird (common coot) (Selim et al., 2017), then observed in domestic birds, including chickens and ducks, as single or co-infected with other respiratory viruses causing severe losses in poultry production (Salaheldin et al., 2018; Yehia et al., 2018; Shehata et al., 2018; Hassan et al., 2019). The low pathogenic avian influenza (LPAI) H9N2 was first recorded in 2010-2011 belonging to G1 lineage (El-Zoghby et al., 2012). It has immunosuppressive effects that lead to increased opportunism of the infection by other viral diseases as mainly observed in co-infection with H9N2 and I.B viruses (Hassan et al., 2017). In addition, the co-infection with other bacterial infections as Escherichia coli and Haemophilus paragallinarum contribute to increasing the virulence of H9N2 because it has an endoproteases enzyme that cleavage precursor HA proteins (Haghighat-Jahromi et al., 2008; Pan et al., 2012; Hassan et al., 2017).

Infectious bronchitis virus is one of the major significant respiratory infections (Hofstad, 1984). It is primarily recorded was in Egypt during the 1950s (Sheble et al., 1986) that related to the Dutch variant D3128 then D274, Mass, 4/91, and D-08880. (Eid, 1998) Novel endemic genotypes associated with Israeli variant 2 and mass serotypes were 
detected in 2006 (Abdel-Moneim et al., 2006a). In 2011, the Egyptian virus was mutated and classified into variant I and variant II (Abdel-Moneim et al., 2012). Both types of IBV virus circulated in Egypt causing outbreaks despite vaccination programs, which all in all lead to high economic losses (Abd El Rahman et al., 2015; Zanaty et al., 2016). The most severe respiratory disease is Newcastle Disease (ND) that causing high mortality in poultry flocks (OIE, 2012). In Egypt, the NDV was first recorded in 1948 (Daubney and Mansy, 1948) then spread rapidly in Egypt despite vaccination programs (Abdel-Moneim et al., 2006b; El-Bagoury et al., 2015).

Virus isolation is the fundamental diagnostic test for avian respiratory viruses, but it appears to be expensive, slow, and labor-intensive (Suarez et al., 2007). Recently, reverse transcriptase-polymerase chain reaction (RT-PCR) and realtime RT-PCR (rRT-PCR) were rapid diagnostic tests used to detect viral nucleic acid (Spackman et al., 2002).

In the recent year, the respiratory viruses (HPAI (H5N8), H9N2 (LPAI)), IB, NDV has continuous evolution that may be affected on the pathogenicity of the virus and vaccine efficacy as previously recorded (Yehia et al., 2018; Hassan et al., 2017; Zanaty et al., 2016; Abdel-Moneim et al., 2006b). The aim of the current research was to explore the updated status of respiratory disease outbreaks in broiler Egyptian farms during 2020 using rRT-PCR and to study the genetic variation of the selected circulating respiratory viruses.

\section{MATERIALS AND METHODS}

\section{Collection and preparation of specimens}

In the present study, AIV (H5N8 and H9N2), NDV, and IBV were examined on 53 infected poultry flocks exhibiting severe respiratory manifestation and high mortality during 2020. The oropharyngeal swabs were obtained from disease or freshly dead birds in nine provinces of Egypt, including Alexandria, Giza, Dakhalia, El-sharqia, Qualiobia, El-Monofia, El-Behira, Domiat, and Cairo (Table 1, Figure 1). A total of 45 chicken flocks (including 36 broilers, 5 commercial layers, and 4 breeder farms), 2 flocks of turkeys, and 6 duck farms were examined. The samples from each flock were collected by 10-15 swabs were pooled in $2 \mathrm{ml}$ of phosphate buffer saline (OIE, 2014; Naguib et al., 2017).

\section{RNA extraction, identification, and subtyping of viruses}

The QIAamp Viral RNA Mini Kit (Qiagen) was used for the extraction of viral RNA from pooled oropharyngeal swabs following the manufacturer's guidelines, then eluted into $50 \mu$ l of nuclease-free water. All samples were tested for the detection of M gene of influenza type A viruses by rRT-PCR (Fereidouni et al., 2012) using the AgPath Real-time Kit (Ambion) and the real-time PCR step one plus System (Applied Biosystems, Foster City, CA, USA). In the next step, subtyping using rRT-PCR targeted HA and NA genes as described by Hoffmann et al. (2016). In addition, all samples were tested for the detection of NDV, IBV using rRT-PCR target matrix gene of avian paramyxovirus-1 (Wise et al., 2004) then the positive samples were tested for the detection of NDV of genotype VII (velogenic strain) (Moharam et al., 2019). Furthermore, rRT-PCR was used for the detection of IBV RNA as described by (Naguib et al., 2017).

\section{Virus isolation}

The positive samples were isolated into specific pathogen-free (SPF) embryonated chicken eggs (ECEs) aged 10 days old in allantoic fluid with daily observation. The mortalities were recorded and the allantoic sac was collected and tested by rapid slide haemagglutination (HA) test (OIE manual, 2008).

\section{Sequencing of the viral genome}

The HA gene of AI (H5, H9) and HVRs of S1 gene of IBV amplification were examined by PCR using specific primers as described by Hoper et al. (2009), Selim et al. (2013) and Naguib et al. (2015), and high fidelity Phusion ${ }^{\circledR}$ DNA polymerase (Thermo Fisher Scientific, MA, USA). Using QIAquick Gel Extraction Kit, the PCR products were separated and purified from the gel (Qiagen).

Cycle sequencing reactions have been performed on PCR products using BigDye Terminator v3.1 Cycle Sequencing Kit (Applied Biosystems). Using centri-sep columns (Macherey-Nagel GmbH \& Co.) the sequence products were purified then sequenced on an ABI PRISM3130 Genetic Analyzer (Life Technologies). For each sequence, the blast search was performed (http://www.ncbi.nlm.nih.gov/BLAST). Mutation and phylogenetic analysis were conducted using MEGA version 6 program using a bootstrap of 1000 trials of the Clustal W alignment algorithm (Tamura et al., 2013). The amino acid identity was analyzed using DNA star software (DNAStar, Madison, WI). Then, it was published by the national center for Biotechnology Information with the accession number provided in Table 2. 
Table 1. Epidemiological data results of PCR of collected samples of collected samples

\begin{tabular}{|c|c|c|c|c|c|}
\hline Number & Governorates & Species & Breeds & Age (day) & Results \\
\hline 1 & El-Dakhlia & Chicken & Broiler & 20 & HPAIV(H5N8) \\
\hline 2 & El-Dakhlia & Chicken & Layer & 275 & HPAIV(H5N8) \\
\hline 3 & El-Dakhlia & Chicken & Broiler & 25 & IBV \\
\hline 4 & Giza & Chicken & Breeder & 90 & IBV \\
\hline 5 & Alexandria & Chicken & Broiler & 30 & IBV \\
\hline 6 & Alexandria & Chicken & Layer & 365 & HPAIV(H5N8) \\
\hline 7 & Giza & Chicken & Broiler & 33 & HPAIV(H5N8) \\
\hline 8 & Giza & Chicken & Broiler & 36 & Negative \\
\hline 9 & Giza & Turkey & & 65 & HPAIV(H5N8) \\
\hline 10 & Giza & Chicken & Broiler & 40 & NDV \\
\hline 11 & El-sharqia & Chicken & Broiler & 22 & IBV \\
\hline 12 & El-Monofia & Chicken & Layer & 390 & NDV \\
\hline 13 & El-sharqia & Chicken & Broiler & 15 & LPAIV(H9N2) \\
\hline 14 & El-Dakhlia & Chicken & Breeder & 392 & IBV \\
\hline 15 & Cairo & Chicken & Broiler & 23 & IBV \\
\hline 16 & Cairo & Chicken & Broiler & 34 & IBV \\
\hline 17 & Domiat & Chicken & Broiler & 42 & HPAIV(H5N8) \\
\hline 18 & El-Dakhlia & Chicken & Broiler & 36 & IBV \\
\hline 19 & El-Dakhlia & Chicken & Layer & 120 & IBV \\
\hline 20 & El-Dakhlia & Chicken & Broiler & 22 & HPAIV(H5N8) \\
\hline 21 & El-Behira & Chicken & Breedrer & 196 & Negative \\
\hline 22 & Giza & Chicken & Broiler & 20 & IBV \\
\hline 23 & Beni-suief & Turkey & & 60 & IBV \\
\hline 24 & Beni-suief & Chicken & Broiler & 25 & IBV \\
\hline 25 & El-Dakhlia & Chicken & Broiler & 15 & IBV \\
\hline 26 & El-sharqia & Chicken & Broiler & 30 & IBV \\
\hline 27 & Domiat & Chicken & Broiler & 15 & IBV \\
\hline 28 & Domiat & Chicken & Layer & 120 & HPAIV(H5N8) \\
\hline 29 & Giza & Chicken & Broiler & 33 & IBV \\
\hline 30 & Alexandria & Chicken & Broiler & 33 & HPAIV(H5N8)+IBV \\
\hline 31 & Alexandria & Chicken & Broiler & 34 & IBV \\
\hline 32 & Cairo & Chicken & Breeder & 95 & IBV \\
\hline 33 & Cairo & Chicken & Broiler & 22 & HPAIV(H5N8) \\
\hline 34 & El-Behira & Duck & & 12 & HPAIV(H5N8) \\
\hline 35 & Domiat & Chicken & Broiler & 18 & Negative \\
\hline 36 & Giza & Duck & & 15 & LPAIV(H9N2) \\
\hline 37 & Giza & Chicken & Broiler & 20 & IBV \\
\hline 38 & Beni-suief & Chicken & Broiler & 15 & HPAIV(H5N8) \\
\hline 39 & El-Monofia & Duck & & 95 & IBV \\
\hline 40 & El-Dakhlia & Chicken & Broiler & 20 & HPAI (H5N8) \\
\hline 41 & Cairo & Chicken & Broiler & 28 & LPAIV(H9N2) \\
\hline 42 & El-sharqia & Duck & & 27 & IBV+LPAIV(H9N2) \\
\hline 43 & El-Monofia & Chicken & Broiler & 18 & NDV \\
\hline 44 & Domiat & Chicken & Broiler & 33 & HPAIV(H5N8) \\
\hline 45 & Domiat & Chicken & Broiler & 23 & IBV+LPAIV(H9N2) \\
\hline 46 & El-sharqia & Duck & & 33 & HPAI (H5N8)+IBV \\
\hline 47 & El-Dakhlia & Chicken & Broiler & 15 & HPAIV(H5N8) \\
\hline 48 & Beni-suief & Chicken & Broiler & 35 & Negative \\
\hline 49 & Cairo & Chicken & Broiler & 34 & HPAIV(H5N8) \\
\hline 50 & El-Behira & Chicken & Broiler & 15 & HPAIV(H5N8) \\
\hline 51 & El-Dakhlia & Duck & & 17 & LPAIV(H9N2) \\
\hline 52 & El-Behira & Chicken & Broiler & 28 & LPAIV(H9N2) \\
\hline 53 & El-sharqia & Chicken & Broiler & 18 & Negative \\
\hline
\end{tabular}

Table 2. Accession number of HA gene of HPAI (H5N8 and H9N2) and S1 gene of infectious bronchitis virus samples

\begin{tabular}{|c|c|c|c|c|}
\hline \multirow{2}{*}{ Number } & \multirow{2}{*}{ Code } & \multicolumn{3}{|c|}{ GenBank Accession number } \\
\hline & & HA(H5) & НА(Н9) & S1 \\
\hline 45 & IBV/EGY/CH/AY1/2020, A/chicken/FW1/2020 & - & MW227513 & MW240842 \\
\hline 30 & IBV/EGY/CH/AY2/2020 A/chicken/Egypt/FN1/2020 & MW227501 & - & MW240843 \\
\hline 3 & IBV/EGY/CH/AY3/2020 & - & - & MW240844 \\
\hline 11 & IBV/EGY/CH/AY4/2020 & - & - & MW240845 \\
\hline 15 & IBV/EGY/CH/AY5/2020 & - & - & MW240846 \\
\hline 39 & IBV/EGY/CH/AY6/2020 & - & - & MW240847 \\
\hline 13 & A/chicken/FW2/2020 & - & MW227514 & - \\
\hline 36 & A/chicken/FW3/2020 & - & MW227515 & - \\
\hline 28 & $\mathrm{~A} /$ chicken/Egypt/FN2/2020 & MW227502 & - & - \\
\hline 40 & A/chicken/Egypt/FN3/2020 & MW227503 & - & - \\
\hline
\end{tabular}




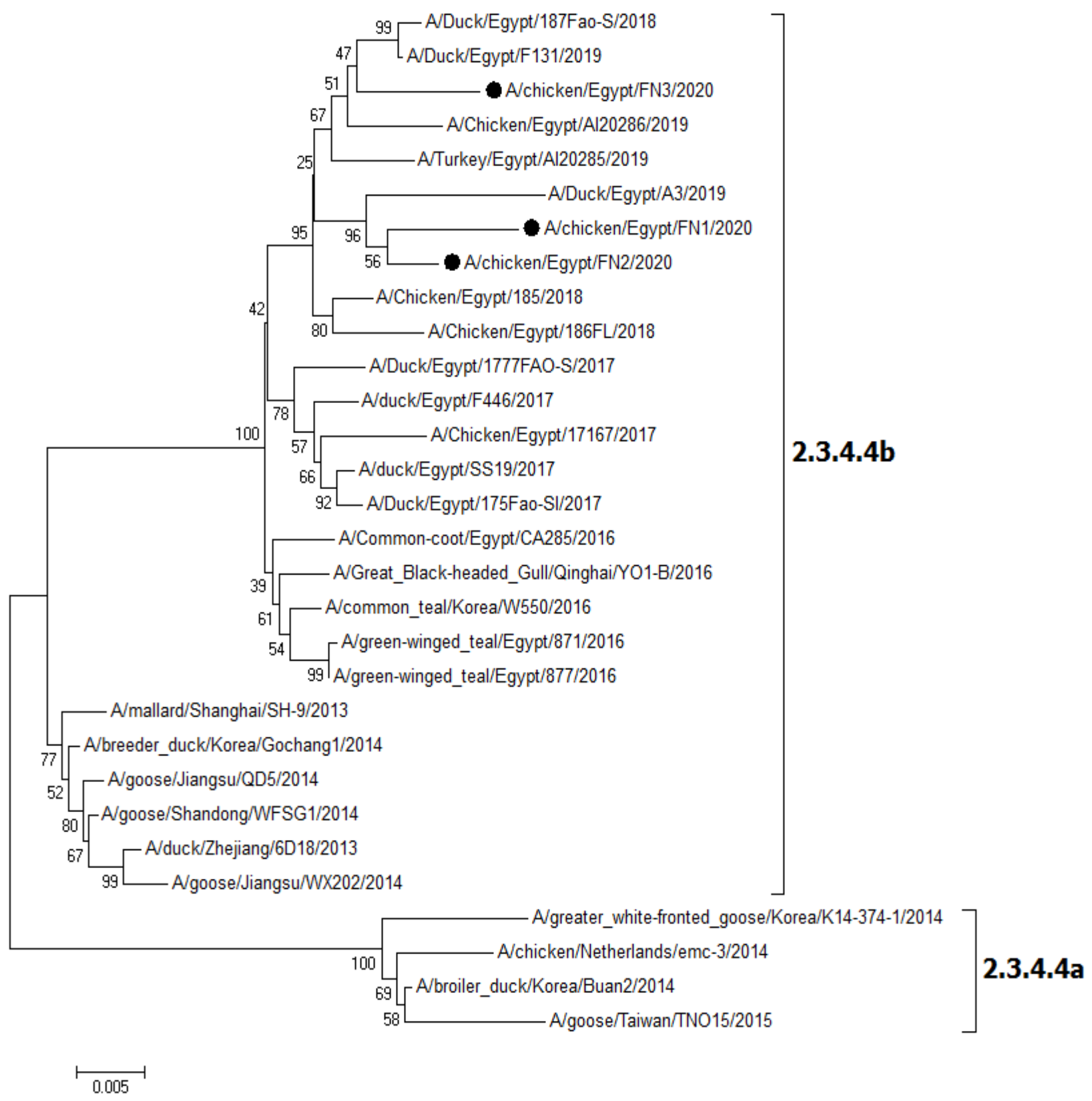

Figure 1. Phylogenetic tree of HA gene of highly pathogenic avian influenza (H5N8)

\section{RESULTS}

\section{Result of real time-PCR}

The findings indicated that 48 samples out of 53 tested samples were positive for RT-PCR for different respiratory diseases (20 samples were positive for IBV, 16 samples for HPAI (H5N8), and 5 samples for LPAI (H9N2), 3 samples for NDV (velogenic strain), 2 samples for HPAI (H5N8) +IBV, and 2 samples for LPAI (H9N2)+I.BV) as mentioned in Table.1.

\section{Genetic characterization of the HPAI (H5N8) Virus HA gene}

Phylogenetic analysis of full HA sequence of four selected AI (H5N8) isolates was named A/ CHICKEN/EGYPT/FN1/2020, A/ CHICKEN/EGYPT/FN2/2020 from co-infected farms and A/ CHICKEN/EGYPT/FN3/202, CHICKEN/EGYPT/FN4/2020 from other infected flocks belonged to clade 2.3.4.4b. They cluster with Egyptian viruses isolated in 2019 making a new subgroup with bootstrapping 95 as shown in Figure 1. They have five nucleotide mutations, compared to A/duck/Egypt/F446/2017, which is different from Egyptian viruses isolated in 2017 and 2018. 
By mutation analysis in antigenic site A, one mutation (T140A) was detected in the A/chicken/Egypt/FN1/2020 and $\mathrm{A} /$ chicken/Egypt/FN2/2020 similar to $\mathrm{A} /$ Chicken/Egypt/186FL/2018. In addition, the receptor-binding site in A/chicken/Egypt/FN3/2020 had R72S was similar to A/Chicken/Egypt/185/2018 and A/Chicken/Egypt/186FL/2018. The S94R was also found only in the A/chicken/Egypt/FN3/2020. The A.A. identity of the current strains and H5N1 vectormune vaccine (A/mute_swan/Hungary/4999/2006), B.E.S.T Vaccine (A/duck/China/E319-2/03) was within the range of 91.9-94\% and H5N2 CEVac Flukem (A/chicken/Mexico/232/1994) was 84.6\% (Figure 2).

\section{Genetic characterization of the H9N2 Virus HA gene}

The phylogenetic analysis of the full HA sequence of three selected AI H9 isolate was named A/ CHICKEN/EGYPT/FW1/2020 from co-infected farms and A/CHICKEN/EGYPT/FW2/2020 and A/ CHICKEN/EGYPT/FW3/2020 from single infected flocks belonged to the A/quail/Hong Kong/G1/97-like virus lineage clustered with group B (Figure 3). The viruses in the current study were closely related to other Egyptian strains with identities of 94.9- 98.2\% (Figure 4). Considering mutation analysis, mutations in S16N, M58K, T121I/V, I134M, T145S, N179T as specific to all Egyptian viruses were recorded and N41G, I75V, V212I, T413N resemble Egyptian viruses in 2018-2019, compared to the A/quail/Hong Kong/G1/97. In addition, R180K/Q was specific for viruses in the present study. The amino acid sequences at the cleavage site of HA contained a low pathogenic RSSR/GLF motif.

\section{Genetic characterization of $S$ gene of infectious bronchitis virus}

The phylogenetic analyses of HVRs of S1 genes of six selected viruses were named IBV/EGY/CH/AY1/2020, IBV/EGY/CH/AY2/2020, and IBV/EGY/CH/AY3/2020 from co-infected flocks and IBV/EGY/CH/AY4/2020, IBV/EGY/CH/AY5/2020, and IBV/EGY/CH/AY6/2020 from single infected flocks. The viruses in this study were clustered to clad GI23 (Egy Var I) (Figure 5).

By mutation analysis of HVRs, the recorded mutation was compared with the reference strain of IBV/CU/4/2014. The HVR1 of three Egyptian viruses (IBV-EGY-CH-AY1-2020, IBV-EGY-CH-AY2-2020, IBV-EGY-CH-AY3-2020) had three A.A. and the other two viruses (IBV-EGY-CH-AY4-2020, IBV-EGY-CH-AY6-2020) had four A.A. However, the IBV-EGY-CH-AY5-2020 had two A.A. The HVRII had 7A.A. mutation in all viruses except IBV-EGY-CH-AY22020, which had six amino acid mutations, and IBV-EGY-CH-AY1-2020, IBV-EGY-CH-AY5-2020 which had four A.A. (Figure 6). The $\mathrm{S} 1$ gene identity of amino acids revealed that viruses in the current study related to vaccine seeds used commonly in Egypt (M41, H120, 4/91) within the range of 68.3-78.8\% (Figure 7).

\begin{tabular}{|c|c|c|c|c|c|c|c|c|c|c|c|c|c|c|c|c|c|c|c|c|c|c|c|c|c|c|c|c|c|c|}
\hline & & & & & & & & & & & & & & & & & & & & & & & & & & & & \\
\hline & 1 & 2 & 3 & 4 & 5 & 6 & & 8 & & & & & & & & & & & & 7 & 9 & 10 & 11 & 12 & 13 & 14 & 15 & 16 & & \\
\hline & & 97.2 & 98.5 & 98.5 & 98.7 & 99.4 & & 99.4 & & & & & & & & & & & & 99.6 & 8.9 & 99.6 & 99.4 & 99.4 & 99.2 & 91.9 & 94.0 & 84.6 & 1 & A-duck-Egypt-F446-2017 \\
\hline \multirow{16}{*}{\multicolumn{2}{|c|}{ 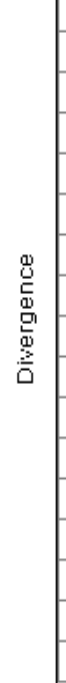 }} & & 97.6 & 97.6 & 96.8 & 97.4 & & 97.6 & & & & & & & & & & & & 97.6 & 74 & 97.6 & 97.7 & 97.7 & 97.4 & 91.7 & 93.8 & 84.9 & 2 & A-broiler-duck-Korea-Buan2-2014 \\
\hline & 1.5 & 2.5 & & 100.0 & 98.1 & 98.7 & 98.9 & 98.7 & 98.1 & & & & & & & & & & & & & 98.9 & 98.7 & 98.7 & 98.5 & 91.7 & 94.0 & 84.6 & 3 & A-goose-Jiangsu-QD5-2014 \\
\hline & 1.5 & 2.5 & 0.0 & & 98.1 & 98.7 & 98.9 & 98.7 & 98.1 & & & & & & & & & & & & & 98.9 & 98.7 & 98.7 & 98.5 & 91.7 & 94.0 & 84.6 & 4 & A-goose-Shandong-WFSG1-2014 \\
\hline & 1.3 & 3.3 & 1.9 & 1.9 & & 98.7 & 99.1 & 98.9 & 98.1 & & & & & & & & & & & & & 8.9 & 98.7 & 98.7 & 98.5 & 91.5 & 93.4 & 84.4 & 5 & A-Chicken-Egypt-17167-2017 \\
\hline & 0.6 & 2.7 & 1.3 & 1.3 & 1.3 & & 99.4 & 99.2 & 99.1 & & & & & & & & & & & & & 9.8 & 99.6 & 99.6 & 99.4 & 91.7 & 93.8 & 84.4 & 6 & A-Common-coot-Egypt-CA285-2016 \\
\hline & 0.4 & 2.5 & 1.1 & 1.1 & 0.9 & 0.6 & & 99.8 & 98.9 & & & & & & & & & & & & & 99.6 & 99.4 & 9.4 & 99.2 & 91.7 & 94.0 & 84.6 & 7 & A-duck-Egypt-SS19-2017 \\
\hline & 0.6 & 2.5 & 1.3 & 1.3 & 1.1 & 0.8 & 0.2 & & 98.7 & & & & & & & & & & & & & 99.4 & 99.2 & 9.2 & 9.1 & 91.7 & 94.0 & 84.6 & 8 & A-Duck-Egypt-175Fao-SI-2017 \\
\hline & 1.1 & 2.7 & 1.9 & 1.9 & 1.9 & 0.9 & 1.1 & 1.3 & & & & & & & & & & & & & & 9.2 & 99.2 & 99.2 & 9.2 & 91.9 & 94.0 & 84.2 & 9 & A-Chicken-Egypt-185-2018 \\
\hline & 0.4 & 2.5 & 1.1 & 1.1 & 1.1 & 0.2 & 0.4 & 0.6 & 0.8 & & & & & & & & & & & & & & 99.8 & 99.8 & 9.6 & 91.9 & 94.0 & 84.6 & 10 & A-Turkey-Egypt-Al20285-2019 \\
\hline & 0.6 & 2.3 & 1.3 & 1.3 & 1.3 & 0.4 & 0.6 & 0.8 & 0.8 & & & & & & & & & & & & & 0.2 & & 00.0 & 9.4 & 91.9 & 94.0 & 84.6 & 11 & A-chicken-Egypt-FN1-2020 \\
\hline & 0.6 & 2.3 & 1.3 & 1.3 & 1.3 & 0.4 & 0.6 & 0.8 & 0.8 & & & & & & & & & & & & & 0.2 & 0.0 & & 9.4 & 91.9 & 94.0 & 84.6 & 12 & A-chicken-Egypt-FN2-2020 \\
\hline & 0.8 & 2.7 & 1.5 & 1.5 & 1.5 & 0.6 & 0.8 & 0.9 & 0.8 & & & & & & & & & & & & & 0.4 & 0.6 & 0.6 & & 91.9 & 94.0 & 84.6 & 13 & A-chicken-Egypt-FN3-2020 \\
\hline & 8.2 & 8.4 & 8.4 & 8.4 & 8.6 & 8.4 & 8.4 & 8.4 & 8.2 & & & & & & & & & & & & & 8.2 & 8.2 & 8.2 & 8.2 & & 97.0 & 87.4 & 14 & A-mute-swan-Hungary-4999-H5N1-vectoremu \\
\hline & 6.1 & 6.3 & 6.1 & 6.1 & 6.7 & 6.3 & 6.1 & 6.1 & 6.1 & & & & & & & & & & & & & 6.1 & 6.1 & 6.1 & 6.1 & 2.9 & & 87.6 & 15 & A-duck-China-E319-2-03-H5N1-B.E.S.T-Vac \\
\hline & 16.4 & 15.9 & 16.4 & 16.4 & 16.6 & 16.6 & 16.4 & 16.4 & 16.8 & & & & & & & & & & & & & 6.4 & 6.4 & 6.4 & 16.4 & 12.9 & 12.9 & & 16 & A-chicken-Mexico-232-1994-H5N2 \\
\hline & 1 & 2 & 3 & 4 & 5 & 6 & 7 & 8 & 9 & & & & & & & & & & & & & 10 & 11 & 12 & 13 & 14 & 15 & 16 & & \\
\hline
\end{tabular}

Figure 2. Amino acid identities and divergence of HA gene of H5N8 viruses compared to other selected strains and vaccinal strains 


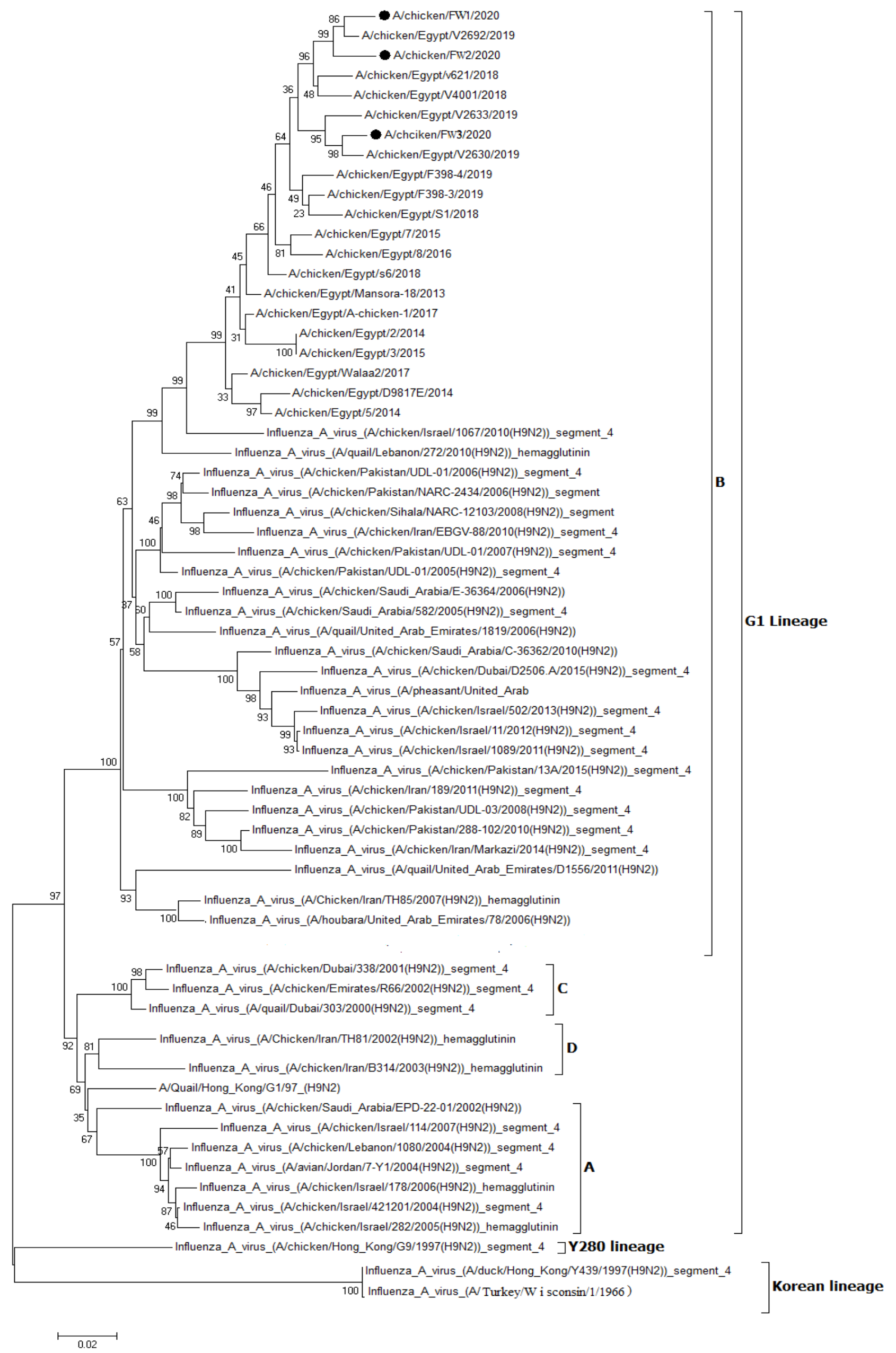

Figure 3. Phylogenetic tree of HA gene of H9N2 


\begin{tabular}{|c|c|c|c|c|c|c|c|c|c|c|c|c|c|c|c|c|c|c|c|c|}
\hline & 1 & 2 & 3 & 4 & 5 & 6 & 7 & 8 & 9 & 10 & 11 & 12 & 13 & 14 & 15 & 16 & 17 & 18 & 19 & \\
\hline 1 & & 85.3 & 88.7 & 92.5 & 92.1 & 92.7 & 93.3 & 91.3 & 90.5 & 87.9 & 88.1 & 87.1 & 89.3 & 88.9 & 88.3 & 87.9 & 87.5 & 87.5 & 88.5 & 1 \\
\hline 2 & 14.8 & & 87.5 & 84.2 & 83.6 & 84.6 & 84.2 & 83.6 & 84.0 & 83.6 & 82.4 & 82.2 & 83.4 & 83.2 & 82.6 & 82.8 & 82.4 & 82.4 & 82.8 & 2 \\
\hline 3 & 10.8 & 12.3 & & 89.5 & 89.3 & 89.1 & 89.5 & 87.7 & 89.3 & 87.1 & 87.1 & 86.3 & 87.5 & 87.5 & 87.3 & 87.1 & 86.5 & 86.5 & 87.9 & 3 \\
\hline 4 & 6.4 & 16.5 & 10.1 & & 98.2 & 93.3 & 93.3 & 91.3 & 90.7 & 87.9 & 88.1 & 87.9 & 89.3 & 88.9 & 88.3 & 87.9 & 87.7 & 87.7 & 88.9 & 4 \\
\hline 5 & 6.9 & 17.2 & 10.3 & 0.8 & & 92.9 & 93.1 & 91.1 & 90.5 & 87.7 & 88.1 & 87.7 & 89.1 & 88.7 & 88.1 & 87.7 & 87.5 & 87.5 & 88.7 & 5 \\
\hline 6 & 6.0 & 15.5 & 10.4 & 5.5 & 6.0 & & 93.1 & 90.9 & 91.3 & 88.3 & 87.9 & 87.1 & 89.3 & 88.7 & 88.1 & 87.9 & 87.3 & 87.3 & 88.7 & 6 \\
\hline 7 & 5.5 & 16.3 & 10.1 & 5.8 & 6.0 & 5.8 & & 92.7 & 92.7 & 89.3 & 89.1 & 89.3 & 90.9 & 90.5 & 89.9 & 89.5 & 89.5 & 89.5 & 90.3 & 7 \\
\hline 8 & 7.8 & 17.2 & 12.2 & 8.0 & 8.2 & 8.3 & 6.4 & & 95.8 & 92.5 & 92.3 & 91.7 & 94.3 & 93.7 & 92.5 & 92.5 & 91.7 & 91.7 & 92.7 & 8 \\
\hline 9 & 8.7 & 16.9 & 10.5 & 8.9 & 9.1 & 7.8 & 6.4 & 3.3 & & 93.7 & 93.3 & 93.1 & 95.8 & 95.2 & 93.9 & 93.7 & 93.1 & 93.1 & 93.7 & 9 \\
\hline 10 & 11.8 & 17.5 & 13.2 & 12.2 & 12.5 & 11.3 & 10.3 & 6.9 & 5.9 & & 98.4 & 95.2 & 96.2 & 96.8 & 97.4 & 97.8 & 94.9 & 94.9 & 97.6 & 10 \\
\hline 11 & 11.5 & 19.0 & 13.2 & 12.0 & 12.0 & 11.8 & 10.6 & 7.1 & 6.4 & 1.4 & & 95.4 & 96.0 & 96.8 & 97.4 & 98.2 & 94.9 & 94.9 & 97.6 & 11 \\
\hline 12 & 12.8 & 19.3 & 14.2 & 12.2 & 12.5 & 12.8 & 10.3 & 7.8 & 6.6 & 4.6 & 4.4 & & 95.2 & 95.8 & 95.6 & 95.6 & 97.6 & 97.6 & 95.8 & 12 \\
\hline 13 & 10.1 & 17.7 & 12.5 & 10.5 & 10.8 & 10.1 & 8.5 & 4.9 & 3.6 & 3.1 & 3.3 & 4.2 & & 97.8 & 96.2 & 96.0 & 94.9 & 94.9 & 96.2 & 13 \\
\hline 14 & 10.6 & 18.0 & 12.7 & 11.0 & 11.3 & 10.8 & 8.9 & 5.5 & 4.4 & 2.7 & 2.7 & 3.8 & 1.4 & & 97.2 & 97.0 & 95.8 & 95.8 & 97.0 & 14 \\
\hline 15 & 11.3 & 18.8 & 12.9 & 11.7 & 12.0 & 11.5 & 9.6 & 6.9 & 5.7 & 2.3 & 2.3 & 4.2 & 3.1 & 2.3 & & 97.8 & 95.6 & 95.6 & 97.8 & 15 \\
\hline 16 & 11.8 & 18.5 & 13.2 & 12.2 & 12.5 & 11.8 & 10.1 & 6.9 & 5.9 & 2.1 & 1.6 & 4.2 & 3.3 & 2.5 & 1.9 & & 95.6 & 95.6 & 97.2 & 16 \\
\hline 17 & 12.3 & 19.0 & 13.9 & 12.5 & 12.7 & 12.5 & 10.1 & 7.8 & 6.6 & 4.8 & 4.8 & 2.1 & 4.4 & 3.8 & 4.2 & 4.2 & & 99.6 & 95.8 & 17 \\
\hline 18 & 12.3 & 19.0 & 13.9 & 12.5 & 12.7 & 12.5 & 10.1 & 7.8 & 6.6 & 4.8 & 4.8 & 2.1 & 4.4 & 3.8 & 4.2 & 4.2 & 0.0 & & 95.8 & 18 \\
\hline \multirow[t]{2}{*}{19} & 11.1 & 18.5 & 12.2 & 11.0 & 11.3 & 10.8 & 9.2 & 6.6 & 5.9 & 2.1 & 2.1 & 4.0 & 3.1 & 2.5 & 1.9 & 2.5 & 4.0 & 4.0 & & 19 \\
\hline & 1 & 2 & 3 & 4 & 5 & 6 & 7 & 8 & 9 & 10 & 11 & 12 & 13 & 14 & 15 & 16 & 17 & 18 & 19 & \\
\hline
\end{tabular}

A-Quail-HongKong-G1-97 A-duck-HongKong-Y439-1997 A-chicken-HongKong-G9-1997 A-chicken-Israel-421201-2004 A-chicken-Israel-178-2006 A-Chicken-Iran-TH81-2002 A-chicken-Dubai-338-2001 A-chicken-Saudi A-chicken-Israel-1067-2010 A-chicken-FW1-2020 A-chicken-FW2-2020 A-chciken-FW3-2020 A-chicken-Egypt-2-2014 A-chicken-Egypt-7-2015 A-chicken-Egypt-v621-2018 A-chicken-Egypt-V4001-2018 A-chicken-Egypt-V2630-2019 A-chicken-Egypt-V2633-2019 A-chicken-Egypt-V2692-2019

Figure 4. Amino acid identities and divergence of HA gene of H9N2 viruses compared to other selected strains and vaccinal strains

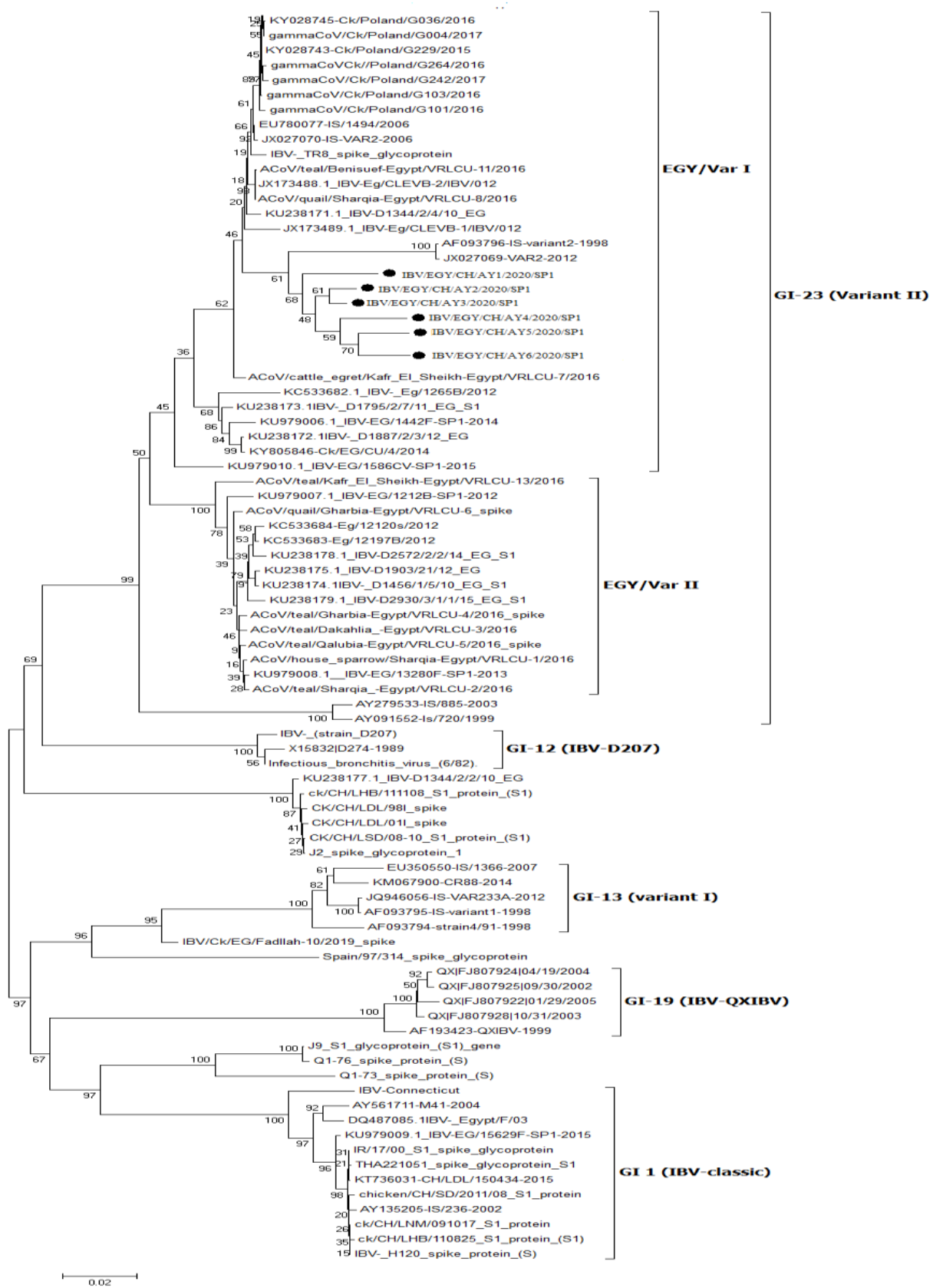

Figure 5. Phylogenetic tree of partial S1 gene of infectious bronchitis virus 


\section{HVRl (60-88)}

cu-4
F859

Eg-CLEVB-2-IBV-012

IBV-Eg-CLEVB-1-IBV-012

IVB-Eg-CLEVB-2-012

IBV-EG-1586CV-SP1-2015

IBV-EG-13280F-SP1-2013

IBV-EG-1212B-SP1-2012

IBV-EG-1442F-SP1-2014

IBV-D2 930-3-1-1-15

IBV-D2572-2-2-14 EG

IBV-D1903-21-12

IBV-D1456-1-5-10

IBV-D1795-2-7-11

IBV-D1887-2-3-12

IBV-D1344-2-4-10

IBV-EGY-CH-AY1-2020

IBV-EGY-CH-AY2-2020

IBV-EGY-CH-AY3-2020

IBV-EGY-CH-AY4-2020

IBV-EGY-CH-AY5-2020

IBV-EGY-CH-AY 6-2020

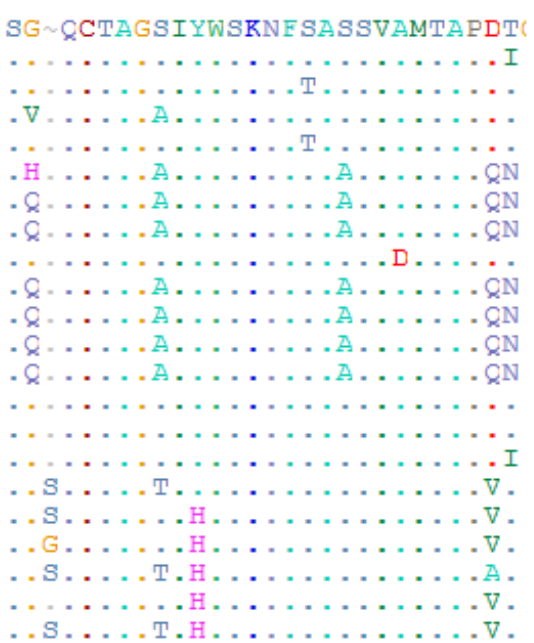

HVR2 (115-149)

YKNGQGSCPLTGLIPQNH IRISAMKNSSI

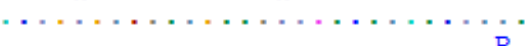

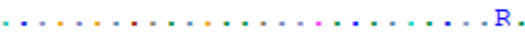

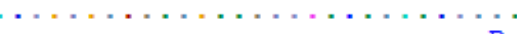

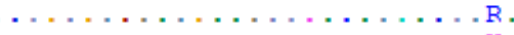

SSS . . . . . . . . . . . . . . . .

.SSS ..........YY.....R.N.

................... R.

.SSS .... M. . . . . R.N.

.S.S.....M....Y....R.N. .

.SSS...............R.N.

. SSS ...... . . YY.....R.N.

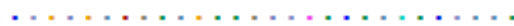

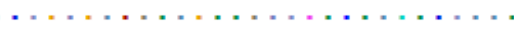

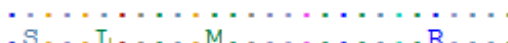

.S... . . . . . . . . . R . .

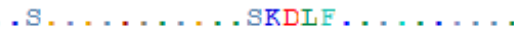

.S......... SKDLF....... .

.H....I..M...HL..V.....H.

.S. . . . . . KD..........

.S......... SKDL..... . . H.

Figure 6. Amino acid mutation in HVRS in $\mathrm{S} 1$ gene of infectious bronchitis virus

\begin{tabular}{|c|c|c|c|c|c|c|c|c|c|c|c|c|c|c|c|c|c|c|c|c|c|}
\hline & & & & & & & & & & Percent & I Identi & & & & & & & & & & \\
\hline & 1 & 2 & 3 & 4 & 5 & 6 & 7 & 8 & 9 & 10 & 11 & 12 & 13 & 14 & 15 & 16 & 17 & 18 & 19 & 20 & \\
\hline 1 & & 90.7 & 71.7 & 75.3 & 71.7 & 72.1 & 73.3 & 71.7 & 72.5 & 72.5 & 80.0 & 68.3 & 75.0 & 72.1 & 76.7 & 74.2 & 75.0 & 78.8 & 76.7 & 73.3 & 1 \\
\hline 2 & 10.1 & & 76.3 & 75.3 & 73.2 & 69.1 & 69.1 & 70.0 & 68.0 & 69.1 & 81.7 & 70.1 & 76.7 & 69.1 & 78.3 & 72.2 & 76.7 & 76.3 & 78.3 & 70.1 & 2 \\
\hline 3 & 34.4 & 25.2 & & 67.0 & 68.8 & 77.1 & 77.9 & 75.0 & 77.1 & 77.5 & 80.0 & 86.2 & 78.3 & 77.1 & 80.0 & 75.4 & 78.3 & 77.1 & 80.0 & 76.7 & 3 \\
\hline 4 & 30.2 & 30.4 & 40.0 & & 67.0 & 64.9 & 64.9 & 66.7 & 67.0 & 67.0 & 70.0 & 69.1 & 70.0 & 66.0 & 71.7 & 70.1 & 71.7 & 72.2 & 71.7 & 68.0 & 4 \\
\hline 5 & 36.2 & 33.9 & 39.6 & 43.7 & & 74.1 & 74.3 & 68.3 & 71.1 & 73.8 & 70.0 & 69.3 & 71.7 & 71.1 & 70.0 & 71.8 & 68.3 & 75.1 & 70.0 & 71.5 & 5 \\
\hline 6 & 33.7 & 36.7 & 27.2 & 43.6 & 32.2 & & 94.0 & 81.7 & 96.8 & 98.0 & 95.0 & 83.8 & 98.3 & 97.5 & 96.7 & 92.8 & 95.0 & 91.7 & 96.7 & 94.2 & 6 \\
\hline 7 & 31.1 & 35.2 & 26.6 & 42.2 & 32.4 & 5.8 & & 80.0 & 90.6 & 93.2 & 88.3 & 81.6 & 91.7 & 91.3 & 90.0 & 88.1 & 88.3 & 87.7 & 90.0 & 89.2 & 7 \\
\hline 8 & 35.7 & 39.2 & 30.5 & 44.1 & 41.1 & 21.2 & 23.6 & & 80.0 & 81.7 & 83.3 & 83.3 & 81.7 & 81.7 & 83.3 & 85.0 & 85.0 & 83.3 & 83.3 & 85.0 & 8 \\
\hline 9 & 32.9 & 38.4 & 27.1 & 39.7 & 34.2 & 2.6 & 8.1 & 23.4 & & 98.2 & 91.7 & 83.8 & 96.7 & 98.6 & 96.7 & 93.5 & 95.0 & 93.5 & 96.7 & 94.9 & 9 \\
\hline 10 & 33.0 & 37.0 & 26.6 & 39.9 & 33.5 & 2.6 & 7.2 & 21.1 & 1.7 & & 93.3 & 84.5 & 98.3 & 98.9 & 98.3 & 93.1 & 96.7 & 93.1 & 98.3 & 94.6 & 10 \\
\hline 11 & 23.3 & 21.1 & 23.3 & 38.4 & 38.4 & 5.2 & 12.8 & 19.1 & 8.9 & 7.0 & & 95.0 & 93.3 & 93.3 & 95.0 & 93.3 & 93.3 & 95.0 & 95.0 & 93.3 & 11 \\
\hline 12 & 40.3 & 36.2 & 14.5 & 36.4 & 38.0 & 19.3 & 22.9 & 18.9 & 18.7 & 18.1 & 5.2 & & 98.3 & 84.1 & 100.0 & 82.3 & 98.3 & 83.8 & 100.0 & 83.8 & 12 \\
\hline 13 & 30.8 & 28.7 & 25.9 & 38.3 & 35.7 & 1.7 & 8.9 & 21.2 & 3.4 & 1.7 & 7.1 & 1.7 & & 98.3 & 98.3 & 96.7 & 96.7 & 98.3 & 98.3 & 96.7 & 13 \\
\hline 14 & 33.6 & 36.6 & 27.1 & 41.6 & 34.9 & 2.1 & 7.7 & 21.1 & 1.3 & 1.3 & 7.0 & 18.7 & 1.7 & & 98.3 & 93.5 & 96.7 & 93.1 & 98.3 & 94.9 & 14 \\
\hline 15 & 28.2 & 26.1 & 23.4 & 35.6 & 38.5 & 3.4 & 10.8 & 18.9 & 3.4 & 1.7 & 5.2 & 0.0 & 1.7 & 1.7 & & 98.3 & 98.3 & 100.0 & 100.0 & 98.3 & 15 \\
\hline 16 & 31.8 & 35.5 & 28.9 & 38.3 & 33.7 & 5.7 & 10.5 & 16.8 & 5.3 & 6.2 & 7.1 & 18.7 & 3.4 & 5.7 & 1.7 & & 100.0 & 94.6 & 98.3 & 97.1 & 16 \\
\hline 17 & 30.8 & 28.7 & 25.9 & 35.6 & 41.6 & 5.3 & 12.9 & 16.8 & 5.2 & 3.4 & 7.1 & 1.7 & 3.4 & 3.4 & 1.7 & 0.0 & & 98.3 & 98.3 & 100.0 & 17 \\
\hline 18 & 25.0 & 29.0 & 26.4 & 35.0 & 29.2 & 7.1 & 11.5 & 18.9 & 5.7 & 6.2 & 5.2 & 17.6 & 1.7 & 6.2 & 0.0 & 4.7 & 1.7 & & 100.0 & 93.9 & 18 \\
\hline 19 & 28.2 & 26.1 & 23.4 & 35.6 & 38.5 & 3.4 & 10.8 & 18.9 & 3.4 & 1.7 & 5.2 & 0.0 & 1.7 & 1.7 & 0.0 & 1.7 & 1.7 & 0.0 & & 98.3 & 19 \\
\hline 20 & 31.7 & 34.9 & 27.7 & 38.0 & 33.5 & 5.3 & 9.6 & 16.8 & 4.8 & 5.7 & 7.1 & 18.1 & 3.4 & 5.3 & 1.7 & 1.3 & 0.0 & 4.4 & 1.7 & & 20 \\
\hline & 1 & 2 & 3 & 4 & 5 & 6 & 7 & 8 & 9 & 10 & 11 & 12 & 13 & 14 & 15 & 16 & 17 & 18 & 19 & 20 & \\
\hline
\end{tabular}

IBV-H120

IBV-M41

IBV-D207

IBV-QXIF

IBV-UK-4-91

IBV-IS-1494-06

IBV-VAR2

IBV-IS-885

IBV-cu-4

IBV-EG-CLEVB-1

IBV-EG-13280F

IBV-EG-1212B

IBV-EG-1442F

IBV-D1344-2-4-10

IBV-EGY-AY1-2020

IBV-EGY-AY2-2020

IBV-EGY-AY3-2020

IBV-EGY-AY4-2020

IBV-EGY-AY5-2020

IBV-EGY-AY6-2020

Figure 7. Amino acid identities and divergence of partial S1 gene of infectious bronchitis virus sequenced viruses compared to other selected strains and vaccinal strains

\section{DISCUSSION}

In Egypt during the last years, the outbreaks due to respiratory disease with high mortality rates have increased and affected poultry production. Many avian viral pathogens were the main cause of this problem (Malik et al., 2004; Roussan et al., 2008). The avian influenza virus (HP, LP), IBV, and NDV were the main detected viruses alone or as coinfected in broiler chickens (Hassan et al., 2016). The current study aimed to identify the incidence of viral respiratory disease in broiler chickens, especially AIV (HP, LP), IBV, and NDV in Egypt during 2020, and study the molecular characterization and evolution of detected viruses.

In the present study, multiple respiratory viruses as single or mixed infections were detected. The AIV subtypes and IBV were recorded in 48 out of 53 farms. Single infection represented 90.6\% (37.7\% I.B, 30.2\% H5N8, 9.4\% I.B and $5.7 \%$ NDV). Furthermore, co-presence of HPAI (H5N8) and IBV, unique detection of these co-infected flocks, and LPAI (H9N2) and IBV, were detected in 3.8\% as previously recorded by El-Shall et al. (2019). The occurrence of IBV and HPAI (H5N8) in Egyptian poultry with a high mortality rate in different vaccinated poultry flocks has previously been reported by a number of researchers (Abdel-Moneim et al., 2006a, Abd El Rahman et al., 2015; Yehia et al., 2020). It was indicated the high incidence of respiratory disease in broiler chicken during 2020 in single infection more than coinfected with other viruses.

In recent years, the avian influenza situation in Egypt has been more complicated due to the detection and circulation of many serotypes, including HPAI (H5N1), HPAI (H5N8), and LPAI (H9N2) (Shehata et al., 2019). The 
phylogenetic analysis of four selected samples of the HA gene of HPAI H5N8 revealed that the four samples were clustered to clad 2.3.4.4.1b as previously recorded by Shehata et al. (2018) and El-Shall et al. (2019) in a new subgroup with characteristic specific mutations due to multiple mutations that may affect the pathogenicity and vaccine efficacy.

Considering mutation analysis, a change was detected in the (T140A) antigenic site A in two samples of $\mathrm{A} /$ chicken/Egypt/FN1/2020 and A/chicken/Egypt/FN2/2020 as previously reported by Yehia et al. (2020) that may be due to excessive use of avian influenza vaccine H5N1 and H5N2. In addition, the R72S in A/chicken/Egypt/FN3/2020 in the receptor-binding site was detected as previously mentioned by Nabil et al. (2020).

The A.A. identity was very low in H5N1 and H5N2 vaccine reaching 91.9-94\% and 84.6\%, respectively, similar to previous studies (Kandeil et al., 2018; Yehia et al., 2020). It explained where the outbreaks occurred in the vaccinated flocks and caused high mortality.

The phylogenetic analysis of three LPAI (H9N2) selected strains revealed that all viruses in the current study belonged to A/quail/Hong Kong/G1/97-like virus lineage clustered with group B as previously recorded (Kareem et al., 2015; El-Shall et al., 2019). Regarding mutation analysis, the LPAI (H9N2) Egyptian viruses had six A.A. mutationspecific. In addition, I75V, N41G, and T413N mutation specific to 2018-2019 Egyptian viruses and 212I mutation that recorded previously had an important effect on the increased pathogenicity of the H9N2 in mice as previously recorded (Yang et al., 2014) that may affect the human transmission of the virus. So, there is a need to conduct further studies to detect the effect of these mutations on the pathogenicity of the virus and vaccine efficacy in poultry.

In the current study, a high number of infected vaccinated flocks with IBV viruses by RT-PCR was recorded. Therefore, partial S1 gene sequencing is important to identify the IBV strains. Furthermore, the partial IBV S1 gene was sequenced, and the analysis revealed that six selected viruses were related to clad GI23 (Egy Var I) as previously indicated (Hassan et al., 2016; Zanaty et al., 2016; Abozeid et al., 2017). The Egyptian strains in this study had low identity percent with vaccine strains H120, M41, 4/91 ranging 68.3-78.8\% that lead to the appearance of infection in vaccinated farms as previously recorded (Abd El Rahman et al., 2015; Sultan et al., 2019). Accordingly, future studies should be conducted to evaluate vaccine efficacy and update the vaccine used to give high protection against newly mutated strains.

The S1 subunit, which comprises three major hypervariable regions (HVRs) in the first 395 amino acids. The HVRs contain major antigenic sites between 38 and 67 amino acids for HVR-1, 97 and 141 amino acids for HVR2, and 274 and 387 amino acids for HVR-3 (Moore et al., 1997). The present study indicated multiple A.A. mutations in the HVR as previously recorded (Hassan et al., 2016; Zanaty et al., 2016; Abozeid et al., 2017) that may be due to widespread use of heterologous vaccine leading to vaccination pressure, which increases the pathogenicity of the virus and changes in tissue tropism (Cavanagh et al., 1992).

\section{CONCLUSION}

Infectious bronchitis virus and highly pathogenic avian influenza (H5N8) are the main causes of respiratory disease in Egyptian flocks alone or co-infected with other viruses with a high mortality rate. Circulation of avian influenza (H5N8) was detected with a mutation in the antigenic site and receptor binding site, low pathogenic avian influenza (H9N2) with a specific mutation, and infectious bronchitis viruses were rapidly evolved in hypervariable regions. The infectious bronchitis virus and highly pathogenic avian influenza H5N8 viruses were distinct from vaccine strains so it is important to surveillance of respiratory disease viruses and study the genetic evolution and its effect on pathogenicity and vaccine efficacy.

\section{DECLARATIONS}

\section{Authors' contribution}

Nahed Yehia contributed to molecular characterization and data analysis and the write-up of the manuscript and Fatma Amer contributed to sample collection and diagnosis.

\section{Competing interests}

The authors have not declared any conflict of interest.

\section{Consent to publish}

All the authors approved and agreed to publish the manuscript.

\section{Ethical consideration}

All authors approved the final draft of the manuscript for publication. Ethical issues (including plagiarism, consent to publish, misconduct, data fabrication and/or falsification, double publication and/or submission, and redundancy) have been checked by the authors. 
Abd El Rahman S, Hoffmann M, Lueschow D, Eladl A, and Hafez HM (2015). Isolation and characterization of new variant strains of infectious bronchitis virus in Northern Egypt. Advanced Animal Veterinary Science, 3(7): 362-371. Available at: https://pubmed.ncbi.nlm.nih.gov/1965995/

Abdel-Moneim AS, Afifi MA, and El-Kady MF (2012). Emergence of a novel genotype of avian infectious bronchitis virus in Egypt. Archive of Virology, 157: 2453-2457. Available at: https://pubmed.ncbi.nlm.nih.gov/22903394/

Abdel-Moneim AS, El-Kady MF, Ladman BS, and Gelb J (2006a). S1 gene sequence analysis of a nephropathogenic strain of avian infectious bronchitis virus in Egypt. Virology Journal, 3: 78. DOI: https://www.doi.org/10.1186/1743-422X-3-78

Abdel-Moneim AS, El-Sawah AA, and Kandil MA (2006b). Characterization of variant strain of Newcastle disease virus in Egypt. BS. Journal of Veterinary Medical Research, 16: 12-17. Available at: https://jvmr.journals.ekb.eg/article 77914.html

Abdelwhab EM and Hafez HM (2011). An overview of the epidemic of highly pathogenic H5N1 avian influenza virus in Egypt: Epidemiology and control challenges. Epidemiology \& Infection, 139(5): 647-657. Available at: https://pubmed.ncbi.nlm.nih.gov/21281550/

Abozeid H, Paldurai A, Khattar SK, Afifi MA, El-Kady MF, El-Deeb AH, and Samal SK (2017). Complete genome sequences of two avian infectious bronchitis viruses isolated in Egypt: Evidence for genetic drift and genetic recombination in the circulating viruses. Infection, Genetics and Evolution, 53: 7-14. Available at: https://pubmed.ncbi.nlm.nih.gov/28495648/

Anis A, AboElkhair M, and Ibrahim M (2017). Characterization of highly pathogenic avian influenza H5N8 virus from Egyptian domestic waterfowl in 2017. avian pathology, 47(4):400-409. Available at: https://pubmed.ncbi.nlm.nih.gov/29701481/

Awad MA, Ali BA, Abd El-Hamid SH, El-Naggar LA, Sediek ME, El-Shall AN, and El-Samahy SH (2016). Epidemiological Studies on H5N1 and H9N2 Avian Influenza Viruses during late 2013 and 2015 in Egypt. Alexandria Journal of Veterinary Sciences, 1(2): 164-173. Available at: https://www.bibliomed.org/?mno=221099

Cavanagh D, Davis PJ, and Cook JKA (1992). Infectious bronchitis virus: evidence for recombination within the Massachusetts serotype. Avian Pathology, 21: 401-408. Available at: https://pubmed.ncbi.nlm.nih.gov/18670955/

Daubney R and Mansy W (1948). The occurrence of Newcastle disease in Egypt. Journal of Comparative Pathology and Therapeutic, 58: 189-200. Available at: https://www.sciencedirect.com/science/article/abs/pii/S0368174248800196

Eid AM. (1998). Infectious bronchitis virus infection in Egypt. Proceedings of the International Symposium on infectious bronchitis and pneumovirus infections in Poultry. Rauischholzhausen, Germany. 145-156.

El-Bagoury GF, Samar F, ElHabbaa AS, and Suzan SE (2015). Isolation, identification, and pathotyping of Newcastle disease virus from chickens in Egyptian. Benha Veterinary Medicine Journal, 29(1): 196-204. Available at: https://bvmj.journals.ekb.eg/article 31819.html

El-Shall NA, Sedeik ME, El-Nahas AF, Abdel-salam RA, and Awad AM (2019). Epidemiological Surveillance of Some Avian Respiratory Viral Diseases in Broiler Chickens. Advanced Journal of Veterinary Science, 61(1): 185-194. Available at: https://www.researchgate.net/publication/332984305_Epidemiological_Surveillance_of_Some_Avian_Respiratory_Viral_Diseases_in_Broiler_C hickens Article History

El-Zoghby EF, Arafa AS, Hassan MK, Aly MM, Selim A, Kilany WH, Selim U, Nasef SM Aggor G, Abdelwhab EM et al. (2012). Isolation of H9N2 avian influenza virus from bobwhite quail (Colinus virginianus) in Egypt. Archives of Virology, 157: 1167-1172. Available at: https://pubmed.ncbi.nlm.nih.gov/22426861/

Fereidouni SR, Harder TC, Gaidet N, Ziller M, Hoffmann B, Hammoumi S, and Starick E (2012). Saving resources: Avian influenza surveillance using pooled swab samples and reduced reaction volumes in real-time RT-PCR. Journal of Virological Methods, 186(1): 119-125. DOI. https://doi.org/10.1016/j.jviromet.2012.08.002

Haghighat-Jahromi M, Asasi K, Nili H, Dadras H, and Shooshtari AH (2008). Coinfection of avian influenza virus (H9N2 subtype) with infectious bronchitis live vaccine. Archives of Virology, 153: 651-655. DOI: https://doi.org/10.1007/s00705-008-0033-X

Hassan KE, Ali A, Dahshan AHM, El-Sawah AA, Shany SAS, and El-Kady MF (2016). Prevalence of avian respiratory viruses in broiler flocks in Egypt. Poultry Science, 95(6): 1271-1280. Available at: https://pubmed.ncbi.nlm.nih.gov/26976895/

Hassan KE, Ali A, Shany SA, and El-Kady (2017). Experimental co-infection of infectious bronchitis and low pathogenic avian influenza H9N2 viruses in commercial broiler chickens. Research in Veterinary Science, 115: 356-362. Available at: https://pubmed.ncbi.nlm.nih.gov/28692924/

Hassan KE, El-Kady MF, El-Sawah AAA, Luttermann C, Parvin R, Shany S, Beer M and Harder T (2019). Respiratory disease due to mixed viral infections in poultry flocks in Egypt between 2017 and 2018: Upsurge of highly pathogenic avian influenza virus subtype H5N8 since (2018). Transboundry and Emerging Disease, 68, 21-36. Available at: https://onlinelibrary.wiley.com/doi/full/10.1111/tbed.13281

Hoffmann B, Hoffmann D, Henritzi D, Beer M, and Harder TC (2016). Riems influenza a typing array (RITA): An RT-qPCR-based low density array for subtyping avian and mammalian influenza a viruses. Scientific Reports, 6: 27211-2721. Available at: https://www.ncbi.nlm.nih.gov/pmc/articles/PMC4891686/

Hofstad MS, Barnes HJ, Calnek BW, Reid WM, and Yoder HW (1984). Diseases of Poultry, Iowa State University Press, Ames, IA. pp. 429-443 Available at: https://www.thepoultrysite.com/publications/diseases-of-poultry/194/infectious-bronchitis-ib

Hoper D, Hoffmann B, and Beer M (2009). Simple, sensitive, and swift sequencing of complete H5N1 avian influenza virus genomes. Journal of Clinical Microbiology, 47: 674-679. Available at: https://www.ncbi.nlm.nih.gov/pmc/articles/PMC2650901/

Kandeil A, Sabir JSM, Abdelaal A, Mattar EH, ElTaweel AN, Sabir MJ, Khalil AA, Webby R, Kayali G, and Ali MA (2018). Efficacy of commercial vaccines against newly emerging avian influenza H5N8 virus in Egypt Scientific Reports, 8: 9697. Available at: https://repository.msa.edu.eg/xmlui/handle/123456789/2270

Kareem EH, Salama AS, Shany AA, Al-Hussien MD, Azza AE, and El-kady M (2015). Prevalence of avian respiratory viruses in broiler flocks in Egypt. Poultry Science, pp. 1-10. Available at: https://pubmed.ncbi.nlm.nih.gov/26976895/

Malik YS, Patnayak DP, and Goyal SM (2004). Detection of three avian respiratory viruses by single-tube multiplex reverse transcription polymerase chain reaction assay. Journal of Veterinary Diagnostic Investigation, 16(3): 244-248. Available at: https://pubmed.ncbi.nlm.nih.gov/15152843/

Moharam I, Abd el Razik A, Sultan H, Ghezlan M, Meseko C, Franzke K, and Grund C (2019). Investigation of suspected Newcastle disease outbreaks in Egypt uncovers a high virus burden in small-scale holdings and the presence of multiple pathogens. Avian Pathology, Pp. 1-31. Available at: https://pubmed.ncbi.nlm.nih.gov/31090444/

Moore KM, Jackwood MW, and Hilt DA (1997). Identification of amino acids involved in a serotype and neutralization specific epitope within the S1 subunit of avian infectious bronchitis virus. Archives of Virology., 142: 2249-2256. Available at: https://pubmed.ncbi.nlm.nih.gov/9672590/

Nabil NM, Erfan AM, Tawakol MM, Haggag NM, Naguib MM, and Samy A (2020). Wild Birds in Live Birds Markets: Potential Reservoirs of Enzootic Avian Influenza Viruses and Antimicrobial Resistant Enterobacteriaceae in Northern Egypt. Pathogens, 9 (3). 169-184. DOI: https://www.doi.org/10.3390/pathogens9030196 
Naguib MM, Arafa AS, El-Kady MF, Selim AA, Gunalan V, Maurer-Stroh S, and Harder TC (2015). Evolutionary trajectories and diagnostic challenges of potentially zoonotic avian influenza viruses H5N1 and H9N2 co-circulating in Egypt. Infection, Genetics, and Evolution, 34: 278291. Available at: https://pubmed.ncbi.nlm.nih.gov/26049044/

Naguib MM, El-Kady MF, Lüschow D, Hassan KE, Arafa AS, El-Zanaty A and Harder TC (2017). New real time and conventional RT-PCRs for updated molecular diagnosis of infectious bronchitis virus infection (IBV) in chickens in Egypt associated with frequent co-infections with avian influenza and Newcastle Disease viruses. Journal of Virological Methods, 245: 19-27. Available at: https://pubmed.ncbi.nlm.nih.gov/28336367/

Pan Q, Liu A, Zhang F, Ling Y, Ou C, Hou N, and He C (2012). Co-infection of broilers with Ornithobacterium rhinotracheale and H9N2 avian influenza virus. BMC Veterinary Research, 8: 104-104. Available at: https://pubmed.ncbi.nlm.nih.gov/22748160/

Radwan MM, Darwish SF, El-Sabagh IM, El-Sanousi AA, and Shalaby MA (2013). Isolation and molecular characterization of Newcastle disease virus genotypes II and VIId in Egypt between 2011 and 2012. Virus Genes, 47(2): 311-316. Available at: https://pubmed.ncbi.nlm.nih.gov/23842723/

Roussan DA, Haddad R, and Khawaldeh G (2008). Molecular survey of avian respiratory pathogens in commercial broiler chicken flocks with respiratory diseases in Jordan. Poultry Science, 87(3): 444-448. Available at: https://pubmed.ncbi.nlm.nih.gov/18281569/

Salaheldin AH, El-Hamid HS, Elbestawy AR, Veits J, Hafez HM, Mettenleiter TC, and Abdelwhab EM (2018). Multiple Introductions of Influenza A(H5N8) Virus into Poultry, Egypt (2017). Emerging Infectious Diseases, 24(5): 943-946. Available at: https://pubmed.ncbi.nlm.nih.gov/29248796/

Samy A and Naguib MM (2018). Avian respiratory coinfection and impact on avian influenza pathogenicity in domestic poultry: Field and experimental findings. Veterinary Sciences, 5(1): 23. Available at: https://www.ncbi.nlm.nih.gov/pmc/articles/PMC5876583/

Sediek ME (2013). Molecular characterization and serotyping of infectious bronchitis virus isolated from broiler chicken farms in Egypt during (2013). International Journal of Agriculture Innovations and Research, 3(1): 286-292. Available at: https://www.sciencedirect.com/science/article/pii/S2314459913000288

Selim AA, Erfan AM, Hagag N, Zanaty A, Samir A, Samy M, Abdelhalim A, Arafa AA, Soliman MA, Shaheen M et al. (2017). Highly pathogenic Avian Influenza virus (H5N8) (clade 2.3.4.4) infection in migratory birds, Egypt. Emerging Infectious Disease Journal, 23(6): 1048-1051. Available at: https://www.ncbi.nlm.nih.gov/pmc/articles/PMC5443452/

Selim K, Arafa A, Hussein A, and El-Sanousi AA (2013). Molecular characterization of infectious bronchitis viruses isolated from broiler and layer chicken farms in Egypt during, 2012. International Journal of Science and Medicine, 1: 102-108. Available at: https://www.sciencedirect.com/science/article/pii/S2314459913000288

Sheble A, Sabry MZ, Davelaar FG, Burger AG, Khafagy AR, Moustafa MM, and Henna M (1986). Present status of infectious bronchitis in Egypt. Journal of the Egyptian Veterinary Medical Association, 46: 393-411.

Shehata AA, Sedeik ME, Elbestawy AR, Zain El-Abideen MA, Ibrahim HH, Kilany WH, and Ali A (2019). Co-infections, genetic, and antigenic relatedness of avian influenza H5N8 and H5N1 viruses in domestic and wild birds in Egypt. Poult Science, 98 (6): 2371-2379. DOI: https://www.doi.org/10.1007/s00705-020-04621-7

Spackman E, Senne DA, Myers TJ, Bulaga LL, Garber LP, Perdue ML, Lohman K, Daum LT, and Suarez DL (2002). Development of a real-time reverse transcriptase PCR assay for type A influenza virus and the avian H5 and H7 hemagglutinin subtypes. Journal of Clinical Microbiology, 40: 3256-3260. Available at: https://pubmed.ncbi.nlm.nih.gov/12202562/

Suarez DL, Das A and Ellis E (2007). Review of rapid molecular diagnostic tools for avian influenza virus. Avian Disease, 51: 201-208. Available at: https://pubmed.ncbi.nlm.nih.gov/17494554/

Sultan HA, Ali A, El Feil WK, Bazid AHI, Zain El-Abideen MA, and Kilany WH (2019). Protective Efficacy of Different Live Attenuated Infectious Bronchitis Virus Vaccination Regimes Against Challenge with IBV Variant-2 Circulating in the Middle East. Frontiers in Veterinary Science, 6: 341. DOI: https://www.doi.org/10.3389/fvets.2019.00341

Tamura K, Stecher G, Peterson D, Filipski A, and Kumar S (2013). MEGA6: Molecular Evolutionary Genetics Analysis version 6.0. Molecular and Biological Evolution, 30: 2725-2729. Available at: https://pubmed.ncbi.nlm.nih.gov/24132122/

Wise MG, Suarez DL, Seal BS, Pedersen JC, Senne DA, King DJ and Spackman E (2004). Development of a real-time reverse-transcription PCR for detection of Newcastle disease virus RNAin clinical samples. Journal of Clinical Microbiology, 42(1): 329-338. Available at: https://pubmed.ncbi.nlm.nih.gov/14715773/

World organization for animal health (OIE) (2015). Manual of Diagnostic Tests and Vaccines for Terrestrial Animals, chapter 2.3.4. Avian influenza. Accessed November 2015. Available at: https://www.oie.int/doc/ged/d12009.pdf

World organization for animal health (OIE) (2008). Update on highly pathogenic avian influenza in animals (type H5 and H7), Pp. 465-476. Available at: https://www.oie.int/fileadmin/Home/eng/Health_standards/tahm/3.03.04_AI.pdf

World organization for animal health (OIE) (2012). Newcastle disease. Manual of Diagnostic Tests and Vaccines for Terrestrial Animals. Chapter, 2.3.14. Available at: https://www.oie.int/ international-standardsetting/terrestrial-manual/access-online.

Yang J, Zhang T, Guo L, Hu Y, Li J, Su H, Xiao Y, Ren X, Dong J, Sun L, Xiao Y, Li L, Yang F, Wang J, Yuan H, and Jin Q (2014). Mutations of novel influenza $\mathrm{A}(\mathrm{H} 10 \mathrm{~N} 8)$ virus in chicken eggs and MDCK cells. Emerging Infectious Disease; 20(9): 1541-1543. Available at: https://pubmed.ncbi.nlm.nih.gov/25148105/

Yehia N, Naguiba MM, Ruiyun Li, Hagag N, El-Husseiny M, Mosaad Z, Nour A, Rabea N, Hasan WM, Hassan MK et al. (2018). Multiple introductions of reassorted highly pathogenic avian influenza viruses (H5N8) clade 2.3.4.4b causing outbreaks in wild birds and poultry in Egypt. Infection, Genetics, and Evolution, 58: 56-65. Available at: https://pubmed.ncbi.nlm.nih.gov/29248796/

Yehia N, Wafaa H, Ahmed S, and Elhusseiny M (2020). Genetic variability of avian influenza virus subtype H5N8 in Egypt in 2017 and 2018. Archives of Virology, 165, 1357-1366. DOI: https://www.doi.org/10.1007/s00705-020-04621-7

Zanaty A, Arafa AS, Hagag N, and El-Kady M (2016). Genotyping and pathotyping of diversified strains of infectious bronchitis viruses circulating in Egypt. World journal of virology, 5(3): 125-134. Available at: https://www.ncbi.nlm.nih.gov/pmc/articles/PMC4981825/ 